\title{
Enlarging Pigmented Eyelid Mass Associated with Remote Pencil Trauma
}

\author{
Paul O. Phelps ${ }^{\mathrm{a}} \quad$ J. Andrew Carlson ${ }^{\mathrm{b}} \quad$ Dale R. Meyer ${ }^{\mathrm{b}}$ \\ ${ }^{a}$ Ophthalmic Plastic Surgery, Lions Eye Institute, Slingerlands, NY, and ${ }^{\mathrm{b}}$ Division of Dermatology and \\ Dermatopathology, Department of Pathology, Albany Medical College, MC-81, Albany, NY, USA
}

\section{Keywords}

Foreign body reaction $\cdot$ Eyelids $\cdot$ Orbit

\begin{abstract}
The authors present a case of a gradually enlarging pigmented mass of the upper eyelid and anterior orbit that was discovered to be the graphite tip of a pencil surrounded by macrophages bearing graphite and fibrous tissue. A 25 -yearold woman with no medical history presented with a gradually enlarging pigmented lesion of her left upper eyelid. She denied any history of previous skin cancer, trauma, or previous surgery. A biopsy was performed. This revealed an encapsulated grayish, pigmented mass within the medial portion of the left upper eyelid and anterior orbit. Within the pigmented cocoon, the graphite core of a pencil ("pencil lead") was identified. Histopathology demonstrated granulomatous inflammation with fibrosis and macrophages.
\end{abstract}

(c) 2017 S. Karger AG, Basel

\section{Introduction}

Pencil injuries to the eye and adnexal tissues have variable presentations within the few reported cases [1-6]. In the skin, pencil "lead" injuries may present anytime between 1.5 and 58 years after implantation of the core [7-

\section{KARGER}

(C) 2017 S. Karger AG, Basel

E-Mail karger@karger.com

www.karger.com/oop
9]. This delayed presentation is thought to be related to granulomatous inflammation which may not occur initially because of the time related to breakdown of the graphite, clay, and waxes contained within the pencil lead [9]. Enlargement of a pigmented mass in patients who often do not have a distinct history of pencil trauma may raise concern for melanoma $[2,7,10]$. Preoperative imaging may be considered to help evaluate the extent and characteristics of some suspicious lesions [11]. However, biopsy may be needed for confirmation of pencil lead injury. When indicated, cautious exploration is an approach to consider.

\section{Case Report}

A 25-year-old woman presented with a gradually enlarging lesion of her left upper eyelid and anterior orbit, near the medial canthus. She reported that the lesion had been present since childhood, and denied any history of skin cancer, trauma, or previous surgery. The patient was concerned because the pigmented mass had increased in size over the past few years, and was now more noticeable. It had a dark gray to purplish hue and was located just superior to the punctum in the left upper eyelid (Fig. 1a). Her vision was not affected and she denied pain. With palpation, the mass was firm and mobile.

Given the progressive nature of the mass, the differential diagnosis included melanoma, vascular lesion, retained foreign body, or other etiology. Biopsy was offered for a definitive diagnosis and

Paul O. Phelps, MD

Ophthalmic Plastic Surgery, Lions Eye Institute

Department of Ophthalmology, Albany Medical College

1220 New Scotland Road, Suite 302, Slingerlands, NY 12159 (USA)

E-Mail pophelps@gmail.com 

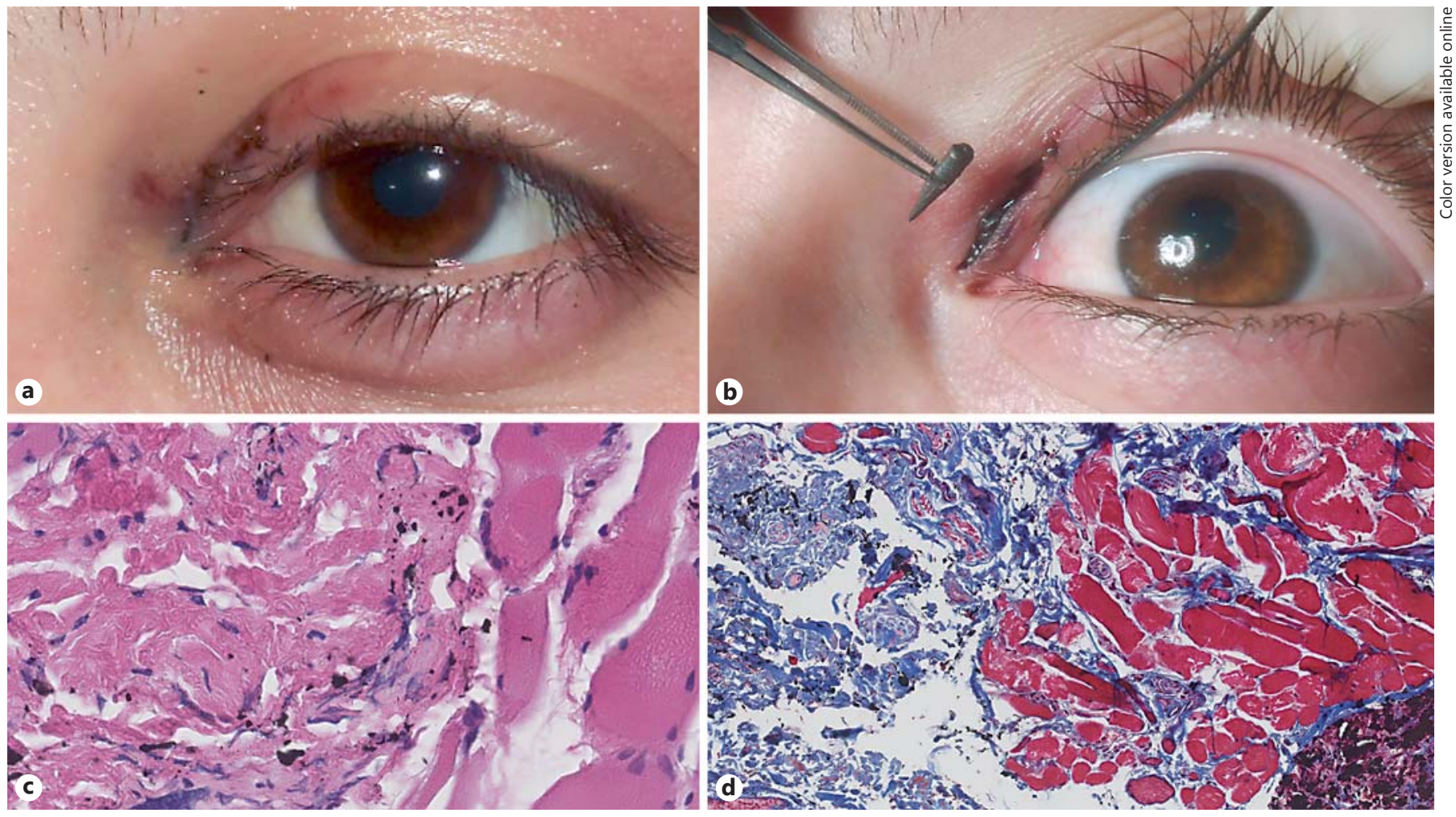

Fig. 1. A 25-year-old woman presented with a gradually enlarging lesion of her left upper eyelid near the medial canthus (a). She reported that the lesion had been present since childhood and had recently increased in size. Biopsy of the lesion revealed the tip of a pencil (b). Histologic examination demonstrated epithelioid his-

tiocytes and giant cells with foreign body pattern containing pigmented material (c, 400× magnification). Trichrome staining demonstrated fibrosis (blue-stained tissue) admixed with pigment-containing macrophages (d, 400× magnification). Orbicularis muscle (red-stained tissue) is also seen.

the patient elected to proceed. Surgical excision was initiated with a probing of the lacrimal system to rule out canalicular involvement. The lesion did appear to be within approximately $2 \mathrm{~mm}$ of the canalicular system. During the surgical excision, we found that there was a well-circumscribed mass with grey pigmentation just below the epidermis, within the orbicularis layer and penetrating through the septum. Removal of some of this cocoon revealed the cylindrical portion of a graphite pencil "lead" (Fig. 1b). Once the core of a pencil was identified, the proximal pigmented tissue was removed. We chose to avoid any extensive dissection of the pigmented tissue once the etiology of the lesion had been determined. Given the sensitive location of the lesion, we considered avoiding damage to surrounding tissues to be the primary goal. The surgical wound was easily closed and the patient was discharged without complication. After surgery, the patient revealed that she did remember being in a pencil fight when she was in grade school; however, she did not remember specifically any pencil injury.

Excisional biopsy demonstrated amorphous deposits of black, pigmented material, striated muscle bundles consistent with orbicularis (Fig. 1c). Regions of fibrosis were confirmed by trichrome staining (Fig. 1d). The extensive deposits of black pigment represented the "cocoon" that surrounded the pencil "lead" tip. Most of the pigmented material was found in macrophages, which ex-

pressed CD68 and CD4. Focally, in areas of fibrosis, scattered lymphocytes, which expressed CD3, CD4, and CD8, could be found. No CD20 (B-cell) lymphocytes were identified. Areas of fibrosis, some vessels, and a small proportion of macrophages expressed CD34. Scattered D2-40 dilated lymphatic vessels were present in regions of fibrosis, indicating presence of lymphatic stasis/poor lymphatic drainage [12]. Additionally, melanocyte markers, MART-1 and S-100, did not find evidence of a melanocytic proliferation. Overall, these findings support the presence of smoldering, subclinical fibro-inflammatory changes and progressive accumulation of graphite-containing macrophages, abetted by poor lymphatic drainage.

\section{Discussion}

Interestingly, the pencil tip injury in our case occurred several decades prior to presentation, and was only revealed after specific questioning after surgery. There appears to be a trend of remote pencil trauma only being revealed after surgical intervention $[2,5]$. Clinicians may

Ocul Oncol Pathol 2017;3:310-312

Enlarging Pigmented Eyelid Mass DOI: $10.1159 / 000470913$

Associated with Remote Pencil Trauma 
want to consider graphite granuloma when they see a pigmented mass in the eyelid or anterior orbit.

We may speculate that the delayed enlargement of these masses may be related to time required for graphite, clay, and waxes in the pencil "lead" to break down. Small particles may enter the interstitium and induce a T-helper and macrophage immune-mediated response. A similar mechanism has been previously proposed [9]. Because the pencil tip was found during surgery, we concluded that this lesion was likely benign in nature and did not proceed to more invasive debridement of orbital tissues.

The pigmented nature of the lesion was important in this case because of the concern for melanoma, as has been noted in previous cases $[2,5]$. CT imaging may be considered to evaluate the extent and character of pigmented lesions in the eyelid or anterior orbit. The scan may reveal a focal hyperdense mass. Melanoma may also be hyperdense, but is less well circumscribed and may be isodense or hypodense on CT scan [13]. MRI is not recommended for patients with possible retained graphite foreign bodies [11]. Ultimately, only surgery and histopathologic evaluation will confirm the diagnosis.

Long-standing foreign body granulomas, which show recent changes in size and color, may be considered in the differential diagnosis of pigmented neoplasms of the eye- lid or anterior orbit. Additionally, imaging with a CT scan of the orbits is recommended because it may help guide the clinician to the diagnosis prior to surgical intervention. It is also advisable that any surgery to remove the pencil "lead" in the anterior orbit be conservative given the delicate structures within proximity of the orbit.

\section{Summary}

A gradually enlarging pigmented mass of the left upper eyelid was thought to represent a melanoma or a foreign body. A biopsy revealed a retained pencil tip surrounded by a capsule of fibrous tissue and low-grade inflammation.

\section{Statement of Ethics}

The collection and evaluation of protected patient health information was HIPPA-compliant.

\section{Disclosure Statement}

The authors have no conflicts of interest to declare.

\section{References}

1 Amritanand A, John SS, Philip SS, John D, David S: Unusual case of a graphite foreign body in the anterior chamber. Clin Pract 2011;1:e73.

2 Guy JR, Rao NA: Graphite foreign body of the conjunctiva simulating melanoma. Cornea 1985;4:263-265.

3 Hamanaka N, Ikeda T, Inokuchi N, Shirai S, Uchihori Y: A case of an intraocular foreign body due to graphite pencil lead complicated by endophthalmitis. Ophthalmic Surg Lasers 1999;30:229-231.

4 Han ER, Wee WR, Lee JH, Hyon JY: A case of retained graphite anterior chamber foreign body masquerading as stromal keratitis. Korean J Ophthalmol 2011;25:128-131.
5 Lee BJ, Gupta S, Flint A, Singer TR, Elner VM: Pigmented orbital mass due to remote pencil trauma. Ophthal Plast Reconstr Surg 2012; 28:e67-e68.

6 Philip SS, John D, John SS: Asymptomatic intracorneal graphite deposits following graphite pencil injury. Case Rep Ophthalmol Med 2012;2012:720201.

7 Hatano Y, Asada Y, Komada S, Fujiwara S, Takayasu S: A case of pencil core granuloma with an unusual temporal profile. Dermatology 2000;201:151-153.

8 Aswani VH, Kim SL: Fifty-three years after a pencil puncture wound. Case Rep Dermatol 2015;7:303-305.

9 Terasawa N, Kishimoto S, Kibe Y, Takenaka $\mathrm{H}$, Yasuno H: Graphite foreign body granuloma. Br J Dermatol 1999;141:774-776.
10 Maudgil A, Wagner BE, Rundle P, Rennie IG, Mudhar HS: Ocular surface foreign bodies: novel findings mimicking ocular malignant melanoma. Eye (Lond) 2014;28:1370-1374.

11 Choudhri AF, Patel BJ, Phillips ME, Mills KA, Whitehead MT, Fleming JC: Diamagnetic susceptibility artifact associated with graphite foreign body of the orbit. Ophthal Plast Reconstr Surg 2013;29:e105-e107.

12 Carlson JA: Lymphedema and subclinical lymphostasis (microlymphedema) facilitate cutaneous infection, inflammatory dermatoses, and neoplasia: a locus minoris resistentiae. Clin Dermatol 2014;32:599-615.

13 Goulart CR, Mattei TA, Ramina R: Cerebral melanoma metastases: a critical review on diagnostic methods and therapeutic options. ISRN Surg 2011;2011:276908. 